\title{
INFLUENCE D'UNE PÉRIODE D'ÉCLAIREMENT CONTINU SUR LA PREMIĖRE MATURATION SEXUELLE DE LA TRUITE ARC-EN-CIEL (ONCORHYNCHUS MYKISS).
}

\author{
G. MAISSE, O. NOËL, B. BRETON, L. GOARDON, P.Y. LE BAIL et B. MOUROT
}

INRA, Laboratoire de Physiologie des Poissons, IFR 43, Campus de Beaulieu, 35042 Rennes Cedex, France.

\section{RÉSUMÉ}

Le taux de maturation sexuelle à 2 ans dans un lot de truites arc-en-ciel (Oncorhynchus mykiss) soumises à un éclairement constant depuis la fin du stade de la résorption de la vésicule a été significativement inférieur à celui du lot témoin. Cette inhibition était presque totale chez les femelles, alors que chez les mâles le taux de maturation diminuait de moitié. Le même phénomène a été observé dans un lot de femelles soumises à une période d'éclairement continu du 1 er juillet $0+$ au 1er juillet $1+$. L'analyse des paramètres physiologiques a montré, chez ces femelles, que la vitellogénèse exogène ne démarrait pas pendant l'année $1+$. De plus, il a été confirmé qu'un éclairement continu a un effet favorable sur la croissance des poissons traités, comparée à celle du témoin.

\section{INFLUENCE OF A CONTINUOUS LIGHT PERIOD ON THE FIRST SEXUAL MATURATION OF THE RAINBOW TROUT (ONCORHYNCHUS MYKISS).}

\begin{abstract}
Sexual maturation rate at the age of 2 years was significantly lower in a group of rainbow trout (Oncorhynchus mykiss) under continuous light from the end of the vesicle resorption stage than in the control. This inhibition was almost total in females, while sexual maturation rate was reduced by half in males. The same phenomenon has been observed in a group of females maintained under constant illumination from the first day of $0+$ July to the first day of $1+$ July. The analysis of the physiological parameters has shown that the exogenous vitellogenesis did not start during the $1+$ year. Moreover, it was confirmed that the continuous light exposure has a beneficial effect on the growth rate of the treated fish in comparison with control.
\end{abstract}

\section{INTRODUCTION}

L'évolution récente de la salmoniculture vers des produits de grande taille, souvent transformés, a été rendue possible par la mise au point et le développement des techniques de triplödisation par choc chaud (CHOURROUT, 1980) ou par choc de pression (CHOURROUT, 1984) qui permettent l'obtention de femelles stériles (LINCOLN et SCOTT, 1984). L'utilisation de ces techniques, en élevage de truites arc-en-ciel (Oncorhynchus mykiss), permet aujourd'hui de produire en routine des femelles de 2 ans stériles, mais en a aussi montré les limites : le taux de triploïdie est variable (DIAZ et al., 1993) et dans 
certains cas les performances zootechniques des individus triploïdes sont plus faibles que celles des témoins diploïdes (VIRTANEN et al., 1990). Ce constat nous a incité à rechercher une technique alternative à la triploïdie permettant l'obtention de truites arc-en-ciel de grande taille ne maturant pas pendant leur vie économique ( 2 ans). Les possibilités de sélection d'une souche, avec une première reproduction à 3 ans, existent (BURGER et CHEVASSUS, 1987) mais cette méthode est délicate à mettre en oeuvre dans le contexte piscicole actuel, avec la nécessité de gérer un cheptel de géniteurs à reproduction tardive. A partir des connaissances acquises sur l'effet des variations de la photopériode sur la reproduction des salmonidés (MAISSE et BRETON, 1996), notre choix s'est porté sur l'utilisation d'un éclairement en continu des poissons pendant certaines périodes de leur cycle vital, l'objectif étant l'inhibition du développement gonadique à 2 ans.

\section{MATÉRIEL ET MÉTHODES}

L'ensemble des expériences a été mené dans la Salmoniculture Expérimentale du Laboratoire de Physiologie des Poissons de I'INRA (SEDI), alimentée par l'eau de la retenue du Drennec, sur la rivière Elorn (Bretagne). La taille des bassins, de forme carrée, était adaptée à la taille des poissons : $1 \mathrm{~m}^{2}$ pour les alevins et $4 \mathrm{~m}^{2}$ à partir de 2 grammes de poids moyen. Chaque bassin était couvert et équipé d'un éclairage artificiel (25 watts pour les $1 \mathrm{~m}^{2}, 50$ watts pour les $4 \mathrm{~m}^{2}$ ) dont l'allumage et l'extinction étaient programmés individuellement. La photopériode naturelle a été reconstituée dans les bassins des lots témoins suivant une programmation hebdomadaire. Les truites arc-en-ciel provenaient d'une souche à reproduction automnale entretenue à la SEDI. L'effectif par bassin ( 2 bassins par lot) était réduit périodiquement, sans tri, afin de ne conserver que 60 (expérience $n^{\circ} 1$ ), 50 (expérience $n^{\circ} 2$ ) et 45 (expérience $n^{\circ} 3$ ) poissons par bassin, à partir du mois de juiliet de la seconde année. Les truites étaient nourries avec un aliment commercial, suivant la table de rationnement établie par le fabricant. Le taux de maturation sexuelle à l'automne $1+$ a été calculé après contrôle de chaque individu, par pression des flancs, de début octobre à fin janvier, puis, après sacrifice au début de l'année 2+ et par observation directe des gonades des poissons n'ayant pas donné de gamètes. Les expériences $n^{\circ} 1$ et $n^{\circ} 2$ ont été menées sur des groupes de poissons des deux sexes. L'expérience $n^{\circ} 3$ a porté sur des femelles uniquement, descendantes de néomâles, génétiquement femelles.

Dans l'expérience $n^{\circ} 1$, les poissons étaient soumis à un éclairement constant dès la fin de la résorption de la vésicule vitelline (FRVV), le 1er janvier 0+ (lot 11) ou à partir du 1 er avril $0+$ (lot 12), jusqu'à janvier $2+$. Dans l'expérience $n^{\circ} 2$, l'éclairement constant a débuté le 1er décembre 0+ (lot 21) ou le 1er mars 1+ (lot 22). Dans ces deux expériences, en dehors des périodes d'éclairement continu, la photopériode naturelle était appliquée. Pour chaque expérience, un lot témoin (lot 1) était mis sous photopériode naturelle pendant toute la durée de l'élevage.

\section{Dans l'expérience $n^{\circ} 3$, les femelles étaient soumises aux photopériodes suivantes:}

- lot témoin : photopériode naturelle pendant la totalité de l'expérience,

- lot 31 : éclairement constant (24L) de la FRVV au 1er juillet $0+$, puis photopériode naturelle,

- lot 32 : photopériode naturelle de la FRVV au 1er juillet $0+$, puis $24 \mathrm{~L}$ jusqu'au 1er janvier $1+$, puis photopériode naturelle,

- lot 33 : photopériode naturelle de la FRVV au 1er juillet $0+$, puis $24 \mathrm{~L}$ jusqu'au 1er juillet $1+$, puis photopériode naturelle,

- lot $34: 24 \mathrm{~L}$ pendant la totalité de l'année $0+$, puis photopériode naturelle. 
Dans la troisième expérience, les paramètres suivants ont été considérés pour chaque bassin :

- le poids moyen à $6,9,12$ et 15 mois, le coefficient de condition ( $K=100 \times \mathrm{P} / \mathrm{L}^{3}$ où $P$ est le poids en gramme et $L$ la longueur totale en centimètre) et les rapports adiposomatique (RAS $=100 \times$ [poids des graisses péristomacales]/[poids du corps éviscéré]) et hépatosomatique (RHS = [poids du foie]/[poids du corps éviscéré]) à 15 mois (mars 1+),

- le poids moyen et le rapport gonadosomatique (RGS $=100 \times$ [poids des gonades] [poids du corps éviscéré]) des poissons n'ayant pas encore ovulé en janvier $2+$ (ensemble des individus),

- le RGS et les niveaux plasmatiques de vitellogénine (Vtg) (dosage ELISA, MOUROT et LE BAIL, 1995) et d'oestradiol-17ß (E2) (dosage radio-immunologique, FOSTIER et al., 1978) en juillet $1+$ (échantillon $n=10$ ),

- la cinétique d'ovulation dans chaque bassin, obtenue par le contrôle de chaque femelle, toutes les deux semaines, à partir du 24 octobre $1+$.

L'analyse statistique a été réalisée à l'aide des tests suivants :

- Chi carré pour les taux de maturation après regroupement des réplicats dans les lots,

- tests de Student $t$ et $t$ modifiés pour la comparaison des moyennes.

\section{RÉSULTATS}

Les résultats des expériences $n^{\circ} 1$ et $n^{\circ} 2$ montrent des réponses aux traitements photopériodiques différentes suivant la période d'application et le sexe. Lorsque l'éclairement en continu est appliqué très précocement (lots 11 et 12), le taux de maturation sexuelle à 2 ans est significativement diminué quel que soit le sexe, mais avec un effet beaucoup plus important chez les femelles (Figure 1). Lorsque le traitement est plus tardif (lots 21 et 22), il n'y a plus d'inhibition de la maturation sexuelle des mâles et seul le traitement, débutant le 1er décembre $0+$, provoque une diminution significative de la maturation sexuelle des femelles à 2 ans (Figure 2).

L'expérience $n^{\circ} 3$ montre que seul l'éclairement en continu, appliqué du 1er juillet 0+ au 1er juillet $1+$, a un effet sur la maturation sexuelle des femelles à 2 ans qui est très fortement inhibée (lot 33, Figure 3). Pour les autres lots (31, 32 et 34), le taux de maturation à 2 ans, la date de première ovulation et la cinétique de reproduction (résultats non présentés) ne diffèrent pas significativement de ceux du lot témoin.

Les paramètres physiologiques, mesurés en juillet $1+$, montrent que la vitellogénèse exogène n'a pas commencé à cette date chez les femelles du lot 33 , contrairement à ce qui se passe pour les autres lots : les niveaux circulants d'E2 (Figure 4) et de Vtg (Figure 5) restent bas et le RGS (Figure 6) se maintient à une valeur très faible.

Le suivi de la croissance des différents lots pendant les 15 premiers mois indique un effet de l'éclairement continu (Figure 7). A 6 mois, le poids moyen des lots 31 et 34, sous $24 \mathrm{~L}$, est significativement supérieur à celui des lots sous photopériode naturelle. A 12 mois, le poids moyen du lot 34 , sous $24 \mathrm{~L}$ depuis un an, est très significativement supérieur à ceux des autres lots. Parmi ceux-ci, les lots 32 et 33, sous 24L depuis 6 mois, ont eu une meilleure croissance que le lot témoin. A 15 mois, en mars $1+$, date présumée d'entrée en vitellogénèse, le lot 33 , qui ne maturera pas à 2 ans, a un poids moyen qui le situe au deuxième rang parmi l'ensemble des lots, dont les poids moyens sont supérieurs de $9 \%$ (lot 32) à $29 \%$ (lot 34) à celui du lot témoin. A cette date, il n'existe aucune différence significative entre les lots pour les paramètres K, RAS et RHS (résultats non présentés). 


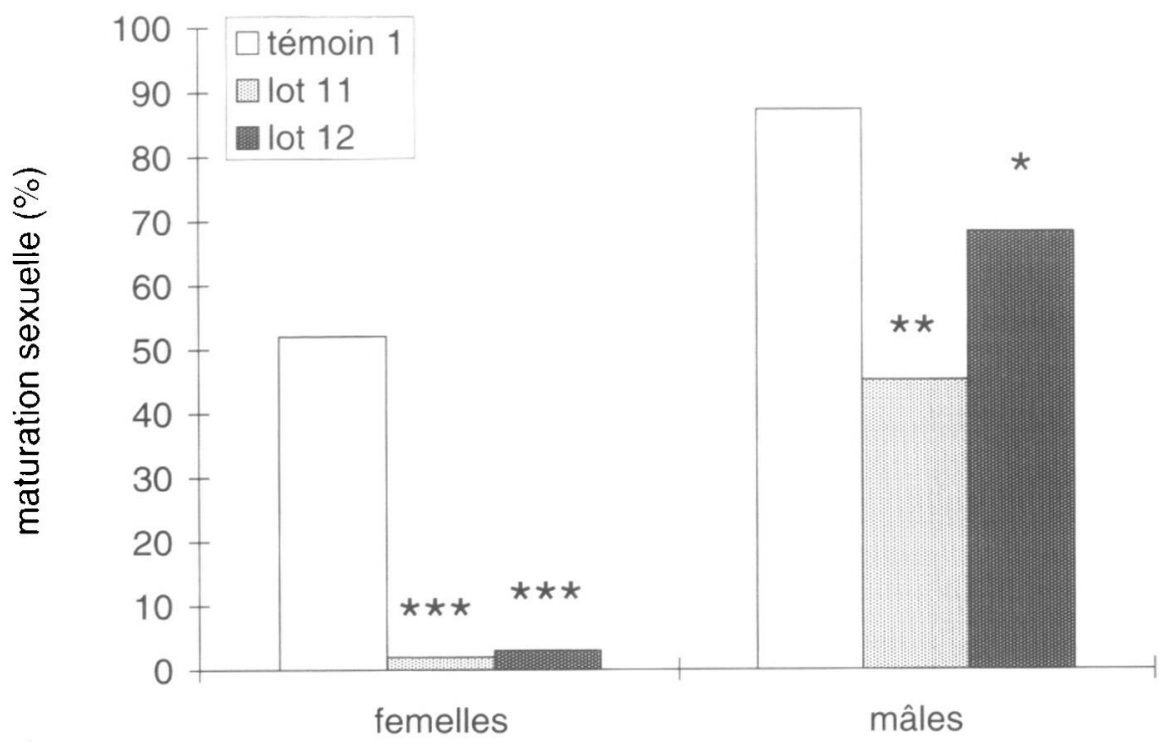

Figure 1

Taux de maturation sexuelle chez les mâles et les femelles des différents lots de truites arc-en-ciel, dans l'expérience $n^{\circ} 1$. La signification des différences par rapport au témoin est indiquée par des astérisques (* pour $p=5 \%$, pour $p=1 \%$, **: pour $p=0,1 \%)$.

\section{Figure 1}

Male and female sexual maturation rate in groups of rainbow trout, in the experiment $n^{\circ} 1$. Asterisks indicate the values which are statistically different from the control.

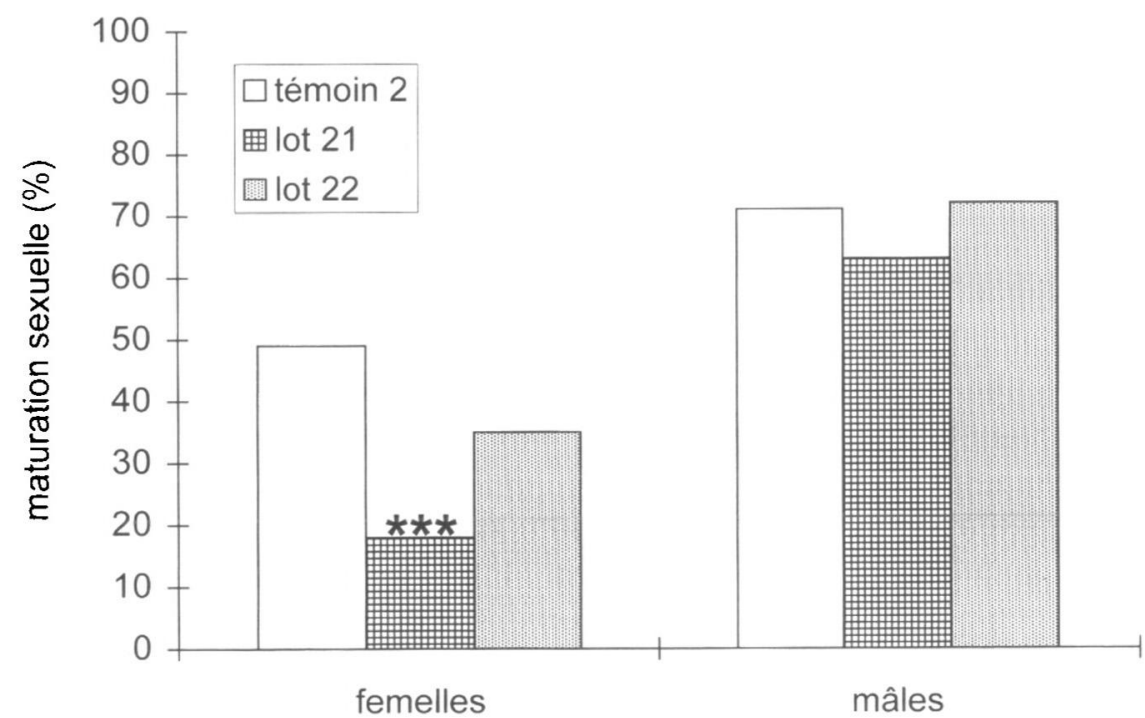

Figure 2

Taux de maturation sexuelle chez les mâles et les femelles des différents lots de truites arc-en-ciel, dans l'expérience $n^{\circ} 2$. La signification des différences par rapport au témoin est indiquée par des astérisques ( pour $p=5 \%$, $*$ pour $p=1 \%, * ;$ pour $p=0,1 \%)$.

\section{Figure 2}

Male and female sexual maturation rate in groups of rainbow trout, in the experiment $n^{\circ} 2$. Asterisks indicate the values which are statistically different from the control. 


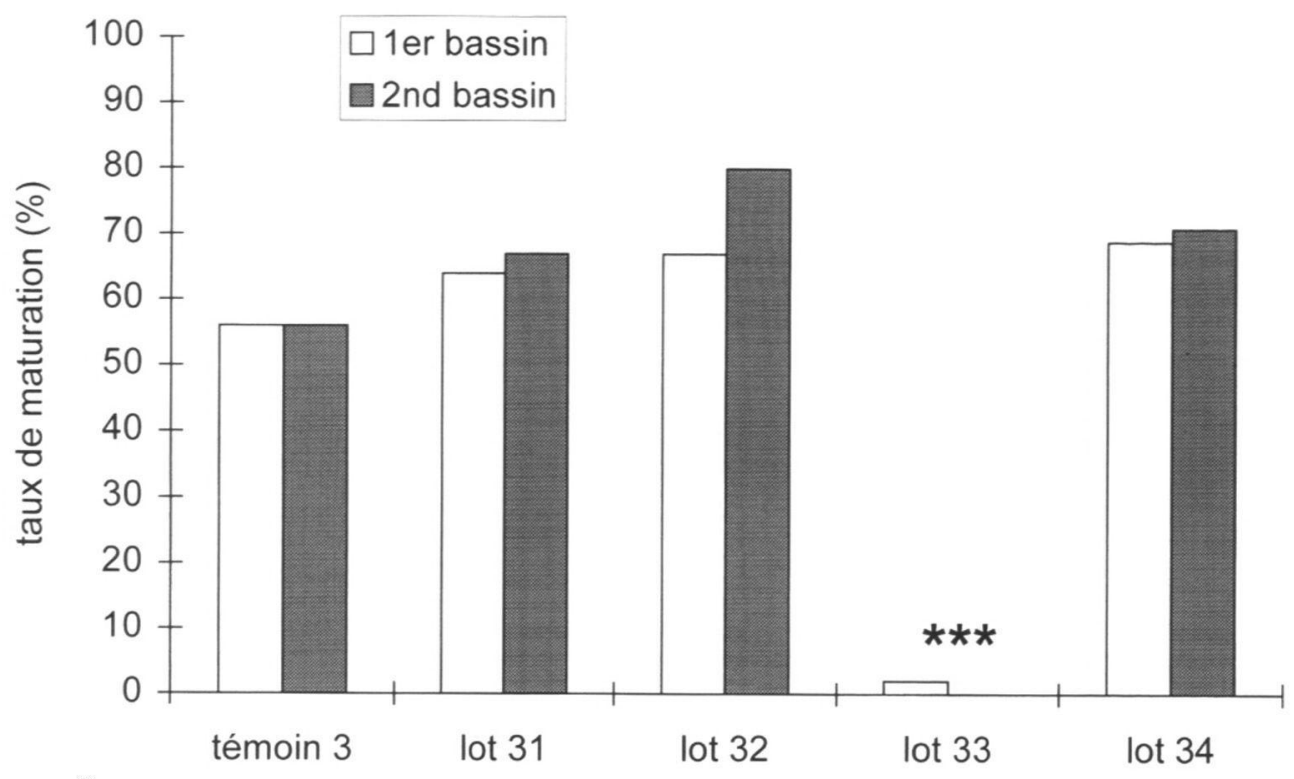

Figure 3

Taux de maturation sexuelle dans les différents lots de truites arc-en-ciel femelles, dans l'expérience $n^{\circ} 3$. La signification des différences par rapport au témoin est indiquée par des astérisques (* pour $p=5 \%, * *$ pour $p=1 \%$, $* * *$ pour $\mathbf{p}=0,1 \%)$.

\section{Figure 3}

Female sexual maturation rate in groups of rainbow trout, in the experiment $n^{\circ} 3$. Asterisks indicate the values which are statistically different from the control.

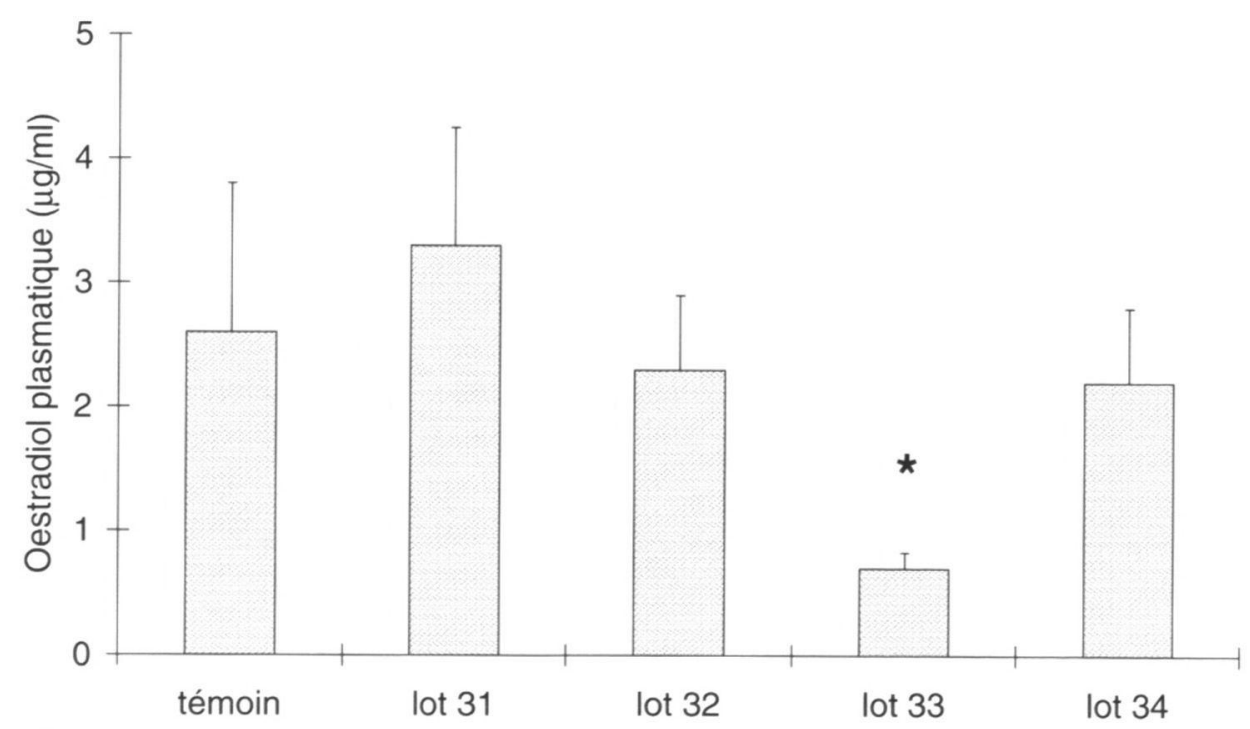

Figure 4

Niveaux moyens d'oestradiol-17 $\beta$ plasmatique $(n=10)$ des différents lots de truites arc-en-ciel femelles, dans l'expérience $n^{\circ} 3$, en juillet $1+$. La signification $(p=5 \%)$ des différences par rapport au témoin est indiquée par un astérisque.

Figure 4

Estradiol-17 $\beta$ mean plasma levels $(n=10)$ in female rainbow trout in the experiment $n^{\circ} 3$, in July $1+$. Asterisk indicates the value which is statistically different $(p=5 \%)$ from the control. 


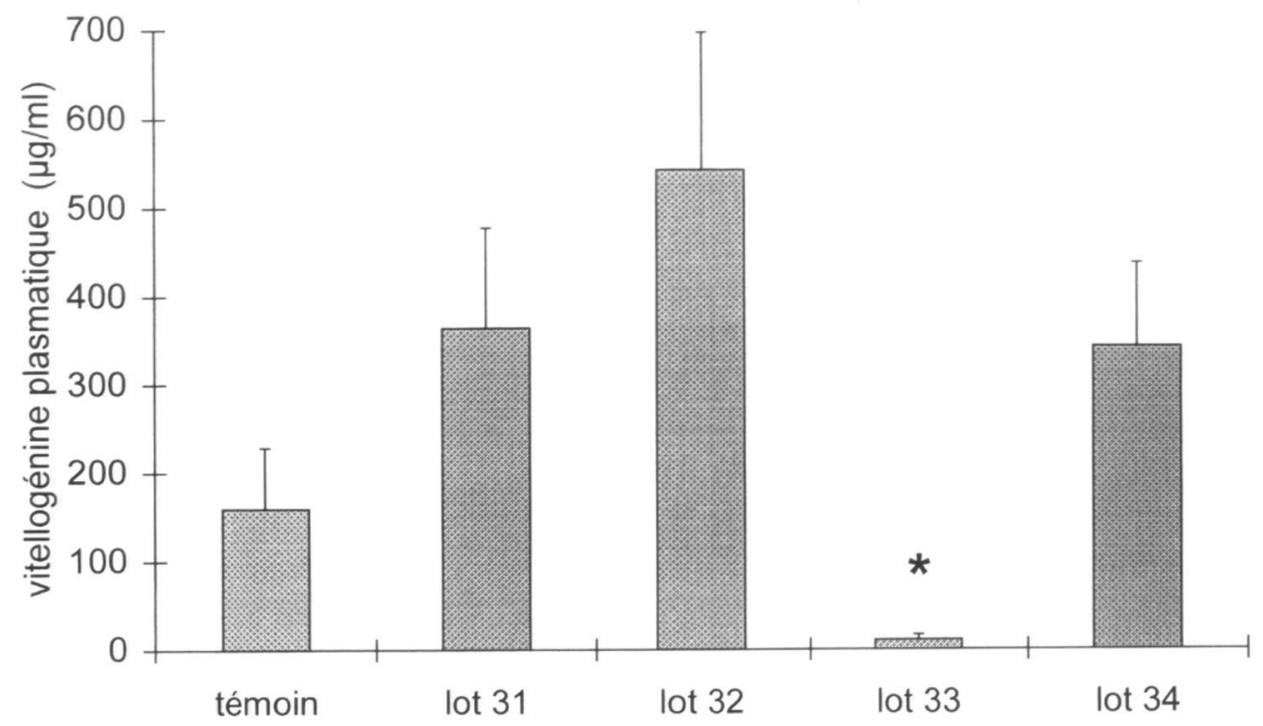

Figure 5

Niveaux moyens de vitellogénine plasmatique $(n=10)$ des différents lots de truites arc-en-ciel femelles, dans l'expérience $n^{\circ} 3$, en juillet $1+$. La signification ( $p=5 \%$ ) des différences par rapport au témoin est indiquée par un astérisque.

\section{Figure 5}

Vitellogenin mean plasma levels $(n=10)$ in female rainbow trout in the experiment $n^{\circ} 3$, in July $1+$. Asterisk indicates the value which is statistically different $(p=5 \%)$ from the control.

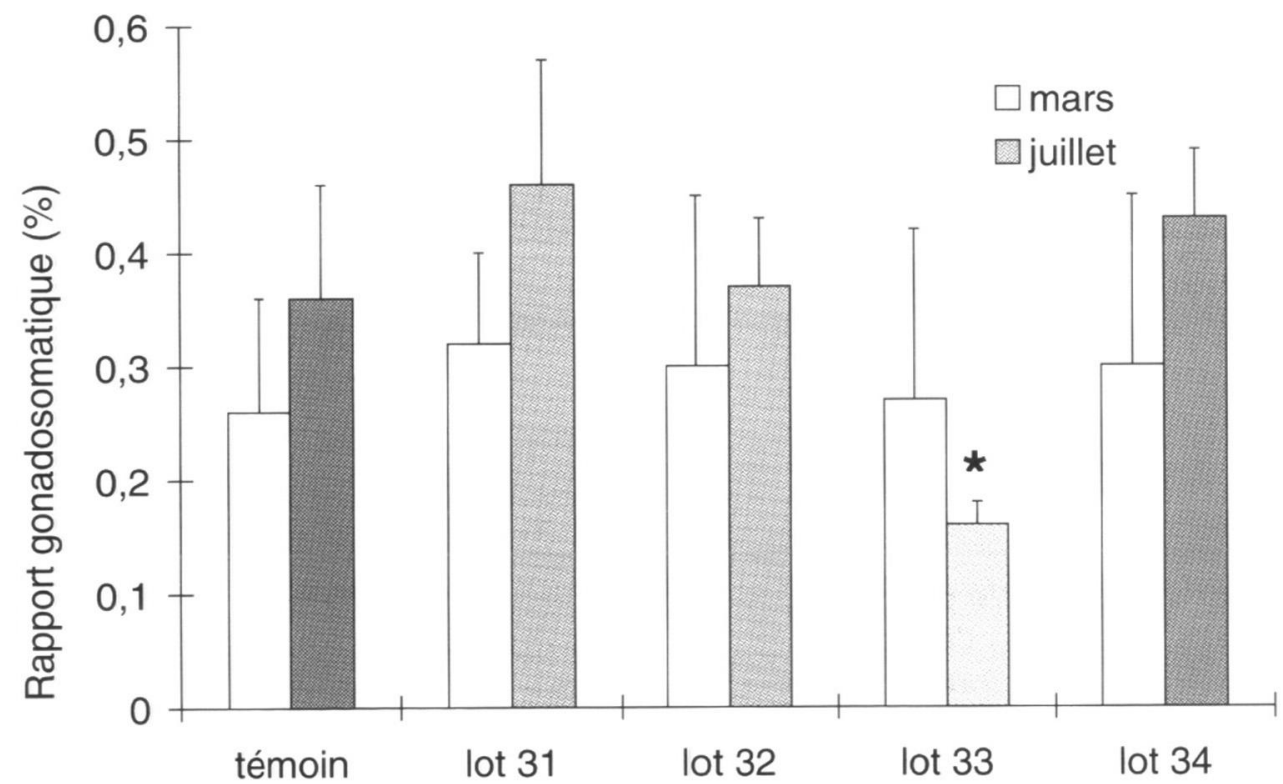

Figure 6

Rapport gonadosomatique chez les femelles $(n=10)$ des différents lots de truites arc-en-ciel, dans l'expérience $n^{\circ} 3$, en mars $1+$ et juillet $1+$. La signification $(p=5 \%)$ des différences par rapport au témoin est indiquée par un astérisque.

\section{Figure 6}

Gonadosomatic index in female $(n=10)$ rainbow trout, in the experiment $n^{\circ} 3$, in March 1+ and in July 1+. Asterisk indicates the value which is statistically different $(p=5 \%)$ from the control. 
En janvier 2+, le RGS, mesuré chez les individus n'ayant pas ovulé, indique qu'ils sont immatures (Figure 8). Le poids moyen des immatures du lot 33 est significativement supérieur aux poids moyens des immatures des autres lots, qui ne diffèrent pas entre eux (Figure 9).

\section{DISCUSSION - CONCLUSION}

L'objectif finalisé de ce travail, qui était d'inhiber la reproduction à 2 ans de la truite arc-en-ciel, a été atteint en grande partie, pour les femelles, par l'application d'un éclairement continu pendant tout l'élevage ou, plus précisément, du 1er juillet $0+$ au 1 er juillet $1+$. Il a été montré que cette inhibition apparaissait très précocement, puisqu'aucun indice sérique de vitellogénèse exogène n'était détectable chez les femelles du lot 33 en juillet $1+$, et n'était pas liée à une croissance moindre des poissons, puisqu'au mois de mars 1+, à la période théorique d'entrée en vitellogénèse exogène de cette souche, le poids moyen du lot traité était plus élevé que celui du lot témoin, les autres paramètres de la croissance étant similaires. Les mâles, quant à eux, subissent les mêmes effets inhibants d'un éclairement continu, mais en moindre proportion que les femelles. Dans la littérature, peu de travaux ont

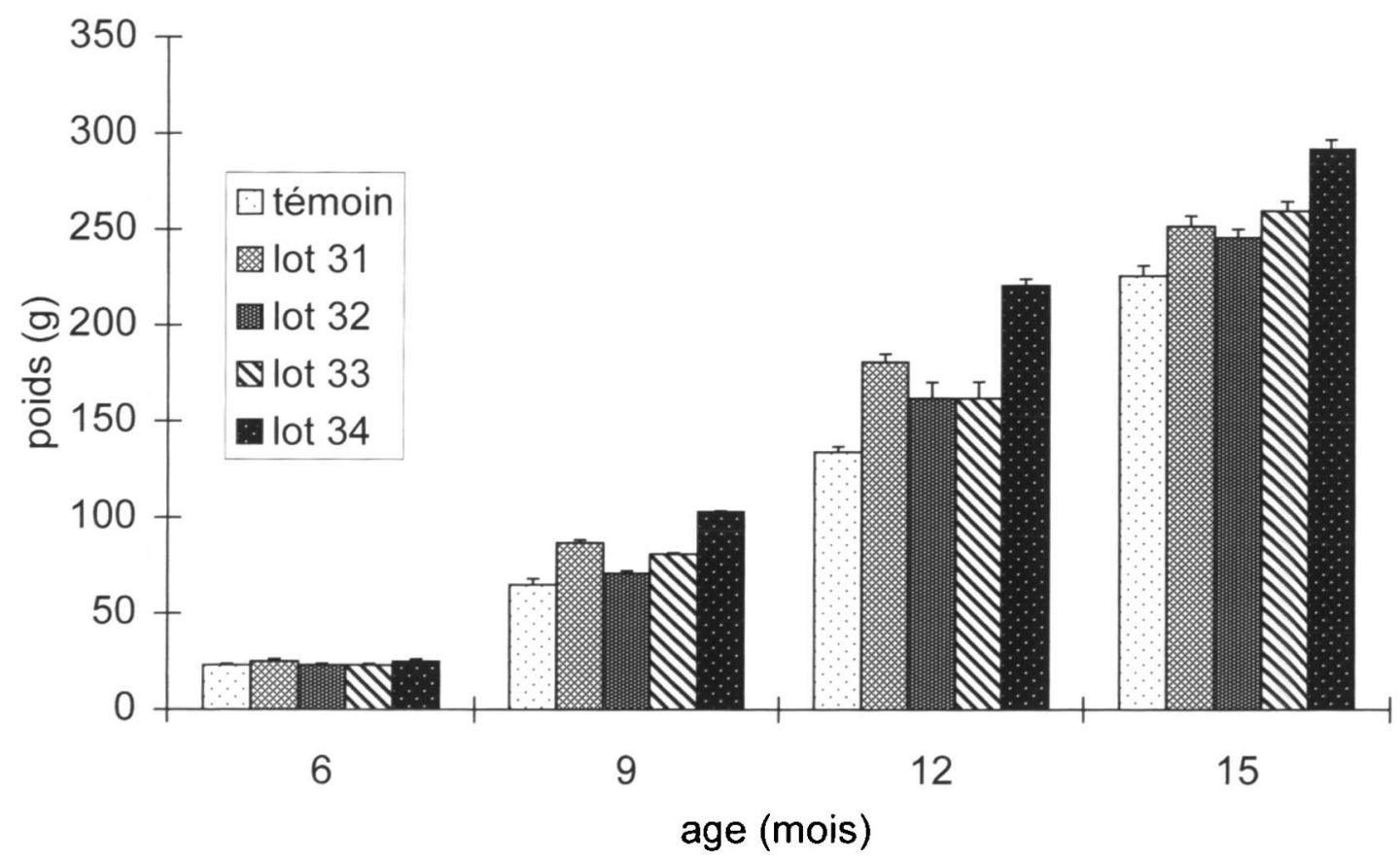

Figure 7

Croissance pondérale des femelles des différents lots de truites arc-en-ciel, dans l'expérience $n^{\circ} 3$. En l'absence de différence significative entre les duplicats de chaque lot, les moyennes présentées ont été calculées à partir de l'ensemble des pesées individuelles dans chaque lot. A 6 mois, le poids moyen des lots 32 et 34 est significativement supérieur à celui des 3 autres lots. A 9, 12 et 15 mois, le poids moyen du lot témoin est significativement inférieur à celui des lots traités.

\section{Figure 7}

Somatic growth of female rainbow trout, in the experiment $n^{\circ} 3$. In the absence of significant difference between replicates, means were calculated from all individual weights in each group. At 6 months of age, the mean weights of groups 32 and 34 are significantly higher than those of the other groups. At 9, 12 and 15 months of age, the control group mean weight is significantly lower than those of the treated groups. 


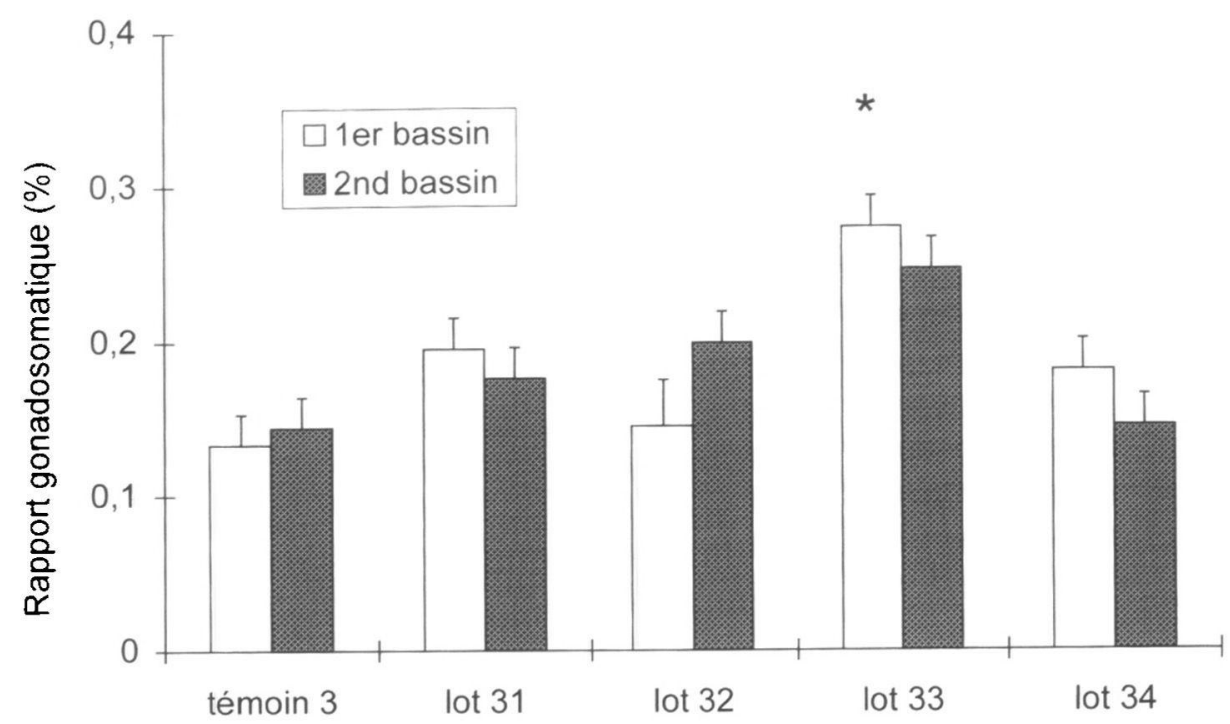

Figure 8

Rapport gonadosomatique chez les femelles non-ovulées des différents lots de truites arc-en-ciel, dans l'expérience $n^{\circ} 3$, en janvier $2+$. La signification ( $p=5 \%$ ) des différences par rapport au témoin est indiquée par un astérisque.

\section{Figure 8}

Gonadosomatic index of non-ovulated female rainbow trout, in the experiment $n^{\circ} 3$, in January $2+$. Asterisks indicate the values which are statistically different $(p=5 \%)$ from the control.

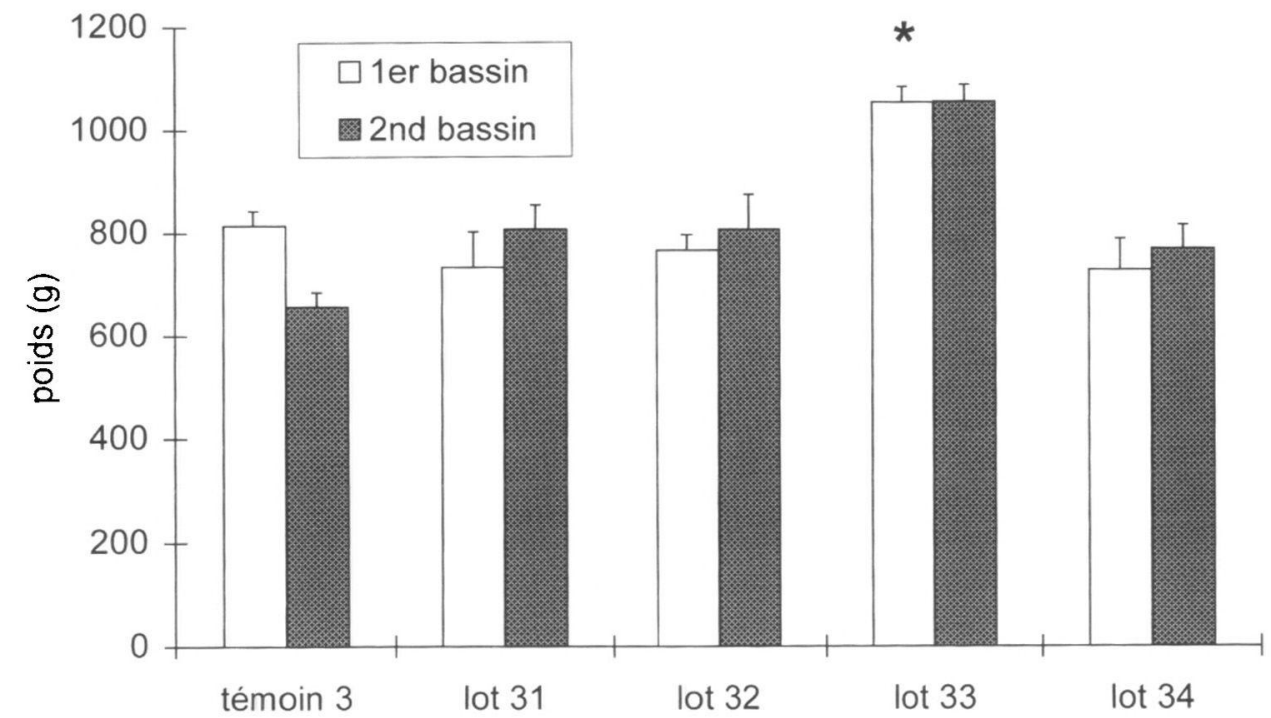

Figure 9

Poids moyen des femelles non-ovulées des différents lots de truites arc-en-ciel, dans l'expérience $n^{\circ} 3$, en janvier $2+$. La signification $(p=5 \%)$ des différences par rapport au témoin est indiquée par un astérisque.

\section{Figure 9}

Mean weight of non-ovulated female rainbow trout, in the experiment $n^{\circ} 3$, in January $2+$. Asterisks indicate the values which are statistically different $(p=5 \%)$ from the control. 
été réalisés afin de mettre en évidence les effets d'un éclairement constant appliqué pendant la première année d'élevage. Chez l'omble de fontaine (Salvelinus fontinalis), PYLE (1969) rapporte une meilleure croissance et n'observe pas d'inhibition de la maturation sexuelle à 2 ans chez des individus soumis à un éclairage constant de mars $0+$ à août $1+$. Chez le saumon atlantique (Salmo salar), SAUNDERS et HENDERSON (1988) ont montré qu'un éclairement constant ou 16 heures de lumière par jour avait un effet bénéfique sur la croissance des juvéniles et que la maturation précoce des mâles était diminuée (respectivement $55 \%$ et $44 \%$ contre $67 \%$ pour le témoin). Les travaux de BERG et al. (1996), bien que portant sur la phase marine de l'élevage du saumon atlantique, se rapprochent des expériences que nous avons réalisées. Ces auteurs montrent qu'un éclairement constant, appliqué immédiatement après transfert en mer, réduit le taux de maturation des mâles, au deuxième hiver de la phase marine, par rapport à un éclairement de 20 heures par jour. Pour les deux régimes photopériodiques, aucune femelle n'a maturé mais, en l'absence de témoin, le rapprochement de cette observation avec nos résultats est impossible.

Les premiers résultats de l'analyse des paramètres physiologiques présentés ici semblent exclure l'hypothèse d'un éventuel stress induit par l'absence de nuit, puisque les performances de croissance des lots sous 24 heures sont supérieures à celles du témoin. Nos résultats semblent plutôt aller dans le sens de l'hypothèse de SUMPTER et al. (1984) concernant l'initiation du premier cycle reproducteur chez la truite arc-en-ciel femelle. Selon cette hypothèse, la mise en place de la première vitellogénèse endogène se ferait un an avant la reproduction. Dans nos expériences, il apparaît que le passage par une période de courte photopériode ( 8 heures de lumière par jour) à l'automne 0+ est toujours associé avec une reproduction à 2 ans. Compte tenu de nos observations et des travaux de RANDALL et al. (1995) qui montrent que les niveaux de mélatonine circulante et la durée de sécrétion par la glande pinéale sont proportionnels à la durée de la nuit, un effet stimulant de la mélatonine dans l'initiation du premier cycle reproducteur n'est pas exclu. L'application d'un traitement par de la mélatonine exogène à des femelles maintenues sous $24 \mathrm{~L}$, dont la sécrétion pinéale est théoriquement inhibée, permettrait de vérifier cette hypothèse. Par ailleurs, l'analyse, en janvier 2+, du RGS des poissons ne s'étant pas reproduits à 2 ans, semble indiquer que les individus du lot 33 ont débuté leur premier cycle sexuel plus précocement que les immatures des autres lots. Cette observation peut être rapprochée des résultats de MACQUARRIE et al. (1979) qui retardent la première reproduction du saumon pink (Oncorhynchus gorbusha) en allongeant la durée du premier cycle photopériodique solaire à 545 jours suivi d'un cycle de 365 jours. L'effet de l'éclairement en continu du 1er juillet $0+$ au 1er juillet 1+ pourrait être assimilé à celui d'un allongement du premier cycle photopériodique solaire à 730 jours. Pour conforter cette interprétation, il serait intéressant de tester les effets d'autres durées constantes d'éclairement ( 16 heures de lumière par exemple) pendant cette période.

En conclusion, d'un point de vue finalisé, l'application d'un éclairement continu du 1er juillet $0+$ au 1 er juillet $1+$, à une population monosexe femelle de truite arc-en-ciel, permet l'inhibition de la maturation sexuelle à 2 ans chez la très grande majorité des individus. Cette technique, qui exerce en outre des effets stimulants sur la croissance, peut être envisagée comme technique de substitution à la technique de triploïdisation.

\section{REMERCIEMENTS}

Les études présentées ont bénéficié d'une aide financière de la Région Bretagne, dans le cadre du programme BRITTA.

Nous remercions les techniciens de la Salmoniculture expérimentale du Drennec qui ont assuré, sous la responsabilité de J. AUBIN, l'élevage des poissons de 1990 à 1997 , L. CARTEAUX, M. HEYDORFF et J.L. ROGER qui ont participé à la collecte des données, ainsi que B. JALABERT et $A$. FOSTIER pour la pertinence de leurs avis. 


\section{BIBLIOGRAPHIE}

BERG A.J., SIGHOLT.T., SELAND A., DANIELSBERG A., 1996. Effect of stocking density, oxygen level, light regime and swimming velocity on the incidence of sexual maturation in adult Atlantic salmon (Salmo salar). Aquaculture, 143, 43-59.

BURGER G., CHEVASSUS B., 1987. Etude des possibilités de sélection de la précocité sexuelle chez la truite arc-en-ciel (Salmo gairdneri R.). Bull. Fr. Pêche Piscic., 307, 102-117.

CHOURROUT D., 1980. Thermal induction of diploid gynogenesis and triploidy in the eggs of the rainbow trout (Salmo gairdneri R.). Reprod. Nutr. Develop., 20, 727-733.

CHOURROUT D., 1984. Pressure-induced retention of second polar body and suppression of first cleavage in rainbow trout : production of all-triploids, all-tetraploids, heterozygous and homozygous diploid gynogenetics. Aquaculture, 36, 111-126.

DIAZ N.F., ITURRA P., VELOSO A., ESTAY F., COLIHUEQUE N., 1993. Physiological factors affecting triploid production in rainbow trout, Oncorhynchus mykiss. Aquaculture, 114, 33-40.

FOSTIER A., WEIL C., TERQUI M., BRETON B., JALABERT B., 1978. Plasma estradiol-17 and gonadotropin during ovulation in rainbow trout (Salmo gairdneri R.). Ann. Biol. Anim. Bioch. Biophys., 18, 929-936.

LINCOLN R.F., SCOTT A.P., 1984. Sexual maturation in triploid rainbow trout Salmo gairdneri Richardson. J. Fish Biol., 25, 385-392.

MACQUARRIE D.W., VANSTONE W.E., MARKERT J.R., 1979. Photoperiod induced off-season spawning of pink salmon (Oncorhynchus gorbusha). Aquaculture, 18, 289-302.

MAISSE G., BRETON B., 1996. Contrôle photopériodique de la saison de reproduction chez les salmonidés. INRA Prod. Anim., 9, 71-77.

MOUROT B., LE BAIL P.Y., 1995. Enzyme-linked immunosorbent assay (ELISA) for rainbow trout (Oncorhynchus mykiss) vitellogenin. J. immunoassay, 16, 365-377.

PYLE E.A., 1969. The effect of constant light or constant darkness on the growth and sexual maturity of brook trout. Fish. Res. Bull. N.Y. State - Div. of Conserv. Dept., 31, 13-19.

RANDALL C.F., BROMAGE N.R., THORPE J.E., MILES M.S., MUIR J.S., 1995. Melatonin rhythms in Atlantic salmon (Salmo salar) maintained under natural and out-of-phase photoperiods. Gen. Comp. Endocrinol., 98, 73-86.

SAUNDERS R.L., HENDERSON E.B., 1988. Effect of constant day length on sexual maturation and growth of Atlantic salmon (Salmo salar) parr. Can. J. Fish. Aquat. Sci., 45, 60-64.

SUMPTER J.P., SCOTT A.P., BAYNES S.M., WITTHAMES P.R., 1984. Early stages of the reproductive cycle in virgin female rainbow trout (Salmo gairdneri Richardson). Aquaculture, 43, 235-242.

VIRTANEN E., FORSMAN L., SUNDBY A., 1990. Triploidy decreases the aerobic swimming capacity of rainbow trout (Salmo gairdneri). Comp. Biochem. Physiol., 96A, 117-121. 